\title{
Autonomous Cooperation Technologies for Achieving Real Time Property and Fault Tolerance in Service Oriented Community System
}

\author{
Naohiro Kaji Khaled Ragab Takanori Ono Kinji Mori \\ Department of Computer Science, Tokyo Institute of Technology \\ 2-12-1 Ookayama Meguro, Tokyo, 152-8552, Japan. \\ Tel: +81-3-5734-2664, Fax: +81-3-5734-2510 \\ E-mail:\{nkaji@mori.,ragab@mori.,nouri@mori.,mori@\}cs.titech.ac.jp
}

\begin{abstract}
The advancement of wireless communication and mobile telecommunication has made mobile commerce possible. In the retail business under the evolving market, the users would like to utilize the appropriate services based on their preference and situation continuously. The service providers need to grasp the current requirements of the majority of the users in the local service area in order to provide the effective service for the current local majority of the service area. However, these local but familiar services cannot be realized through global information services on the Internet.

The Service Oriented Community is proposed in order to satisfy both users' and service providers' requirements. In the Community, the members cooperate with each other in order to get mutual benefits like social community. The users provide their requirements to service providers while utilizing the services, and service providers utilize the users marketing information to provide appropriate services. This marketing information should be collected in the flexible area based on services in real time. Hence flexibility, real-time property, and fault tolerance are required. Here, Time Distance Oriented Information Service System has been proposed to achieve flexibility in collecting the users' marketing information in the area based on services.

In the community system, the key idea is autonomous cooperation among nodes to satisfy the requirements. Here, Autonomous Synchronization Technique and Autonomous Cooperation Technique are proposed to satisfy real time property and fault tolerance respectively. The effectiveness of these technologies is shown at the end.
\end{abstract}

\section{Introduction}

The advancement of wireless communication and mobile telecommunication has made it possible that users access information services with a mobile device anytime and anywhere. Mobile devices have been pervasive. In Japan more than 50 million users access the Internet with mobile phones like I-mode. The market of mobile commerce has been broadened.

Under the evolving market, it has been increasing even more than the requirement for mobile commerce to provide the situation-aware appropriate services. It means not only location-aware but also timely services and the service reflecting the users' preference, which cannot be satisfied through the global information services such as e-commerce on the Internet.

Systems using a service accelerator system and an autonomous decentralized service system to provide personal service have been reported [2,3]. These systems mediate between service providers and users and provide individual user with services based on their profiles. However, cooperation among users based on the local majority in the temporary occasion is not considered. Conventional location awareness systems using mobile terminal provide services based on the users location [4, $5,6]$. These technologies describe a basic concept of service mediation platform, but they assumed that each service is provided to static area. Provision to the dynamic area cannot be realized.

In order to provide and utilize the appropriate services which are timely and reflecting the users' preference in the local area, the mutual cooperation between users and service providers is required, where users provide their preference information and meanwhile service providers utilized the marketing information in the local area at the moment. This mutual cooperation should be done among service providers and users in the dynamic area based on each service and at the moment under the evolving 
market. Therefore flexibility and real-time property is necessary for the system.

Time Distance Oriented Information Service System [7] has been proposed to achieve flexibility in providing the information in the dynamic area based on each service. However, real-time property in collecting the users' marketing information and fault tolerance in providing the services has not been discussed. This paper shows the proposition of Autonomous Synchronization Technique and Autonomous Cooperation Technique to achieve real-time property and fault tolerance respectively.

This paper is structured as follows. Next section presents the application and system requirements. Section 3 discusses Service Oriented Community System. Section 4 exposes our proposed technologies. Section 5 shows the effectiveness of these technologies. Last section concludes the paper.

\section{Requirements}

\subsection{Application Requirements}

\subsubsection{Users Requirements}

The circumstances surrounding users are constantly changing and so are their requirements. Users require the utilization of unknown but appropriate services based on their current situations and preferences, which are provided by the Service Providers within accessible area. For example, the users tend to buy daily necessities near their houses. On the other hand, they tend to buy expensive products at a department store that is situated far from their houses.

The users also would like to cooperate with each other by sharing their requirements and reduce the price of goods that they would like to buy. If many users in the neighborhood temporarily have the same requirement, they make the pressure group to drive down the price.

\subsubsection{Service Providers Requirements}

In the retail business Service Provider (SP) has provided services for the users not in the global but in the local area around it. SP would like to provide the effective service to the users in the local service area. SP would like to grasp the users' marketing information at the moment and to determine the most effective service for them because users in the service area change at every moment by moving and these users' requirement also changes with time.

The service areas differ according to each service. For example, inexpensive goods like bread and vegetables are bought by users near the SP, and expensive goods like electric devices and PC are bought also by users far from the SP. Therefore SP would like to change the area where it grasps the users' marketing information.

\subsection{Service Oriented Community}

The Service Oriented Community is proposed in order to satisfy both the requirements of the users and that of
SP. The Community consists of members such as the users and SP in the specified area based on services.

In the Community, the members cooperate with each other in order to get mutual benefits like social community. The users provide their requirements to SP as well as utilize the services, and SP utilize the users marketing information as well as provide the services.

As the result, SP grasps the current majority requirement in the community by collecting the users' marketing information, and determines the most appropriate service for the users.

\subsection{System Requirements}

For satisfying both users' and SP's requirements, the information system under the evolving environment has to satisfy asked for the following requirements.

\section{Flexibility}

SP would like to provide the effective service for users in the service area based on service. The service areas differ according to each service. Therefore flexibility to change the community area to provide and utilize the information based on service itself is required for the system.

\section{Real-time property}

Users would like to utilize the appropriate service at the moment, and SP would like to grasp the marketing information that consists of the users around SP at the moment. Users around SP are constantly changing because users are moving, and users' requirements change constantly as the current situation changes. Therefore real time property to the dynamic situation is required for the system.

\section{Fault tolerance}

Both the users and the SP require the stable utilization and provision of the services. As the network becomes large under the changing environment, it may include the failure. Therefore fault tolerance is necessary to achieve high service availability for both users and the SP.

\section{Service Oriented Community System}

Service Oriented Community System called Time Distance Oriented Information Service System has been proposed, which achieves flexibility described previously.

\subsection{Time Distance}

Time Distance is a logical distance that is defined by user's physical moving time [7]. The community area which means the user's accessible area based on service cannot be defined by physical distance because user's access time is different based on the physical condition, like a river or some physical obstacles, and the condition like traffic jam even if physical straight distance is the same. Hence, Time Distance has been proposed to measure the community area based on services. 


\subsection{System Architecture}

The system is structured based on Autonomous Decentralized Systems [1]. It consists of the autonomous subsystems; nodes, SP and users with mobile devices.

Figure 1 shows the system architecture. Nodes are base stations of the wireless network. They transmit data between physically neighboring nodes, and broadcast messages to the users within the sphere of the cell. Each node has a time distance table, in which time distances are registered from neighboring nodes.

SP communicates with the physically nearest node and it has its own services that change dynamically in the evolving situation. Each service has the lifetime determined by the SP and is distributed to the users in the appropriate service area based on the lifetime.

The users have a wireless connection to the nearest node. Each user has his preference that contains his demand information.

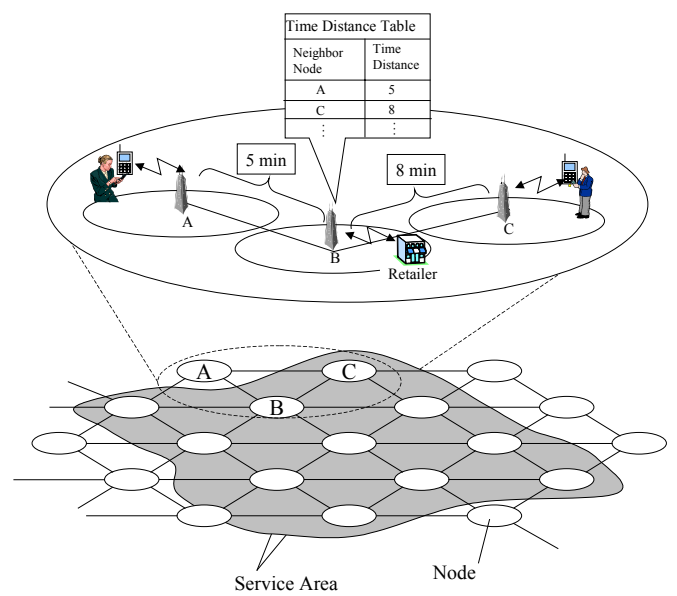

Figure 1: System Architecture

\subsection{Autonomous Information Provision}

Autonomous Information Provision Technology has been proposed to achieve flexibility of the information provision based on services [7]. It makes the request message for collecting users' information provided in the community based on the lifetime of the service.

The request message contains the Content Code (CC), the Service Lifetime (SLT), the Cumulative Time Distance (CTD), Service ID (SID) and the service's information. CC shows that this message is a request message. SLT determines the service area, defined by time distances, of the service that SP can describe based on each service. Each node adds the time distance to CTD. SID shows the unique identifier of the message.

SP sends the request message attached the SLT to the nearest nodes. This request message is propagated in the community of SLT in the following way.

When the node receives the request message directly from $\mathrm{SP}$, it attaches $\mathrm{CTD}=0$ in the message and sends it to all the neighbor nodes. Each node receiving the request message updates CTD by adding the time distance from former node, and autonomously judges whether the request is valid for forwarding or not based on updated CTD. If the CTD is larger than SLT, the node disposes it. Otherwise, the node sends the message to all the neighbor nodes except the sending node.

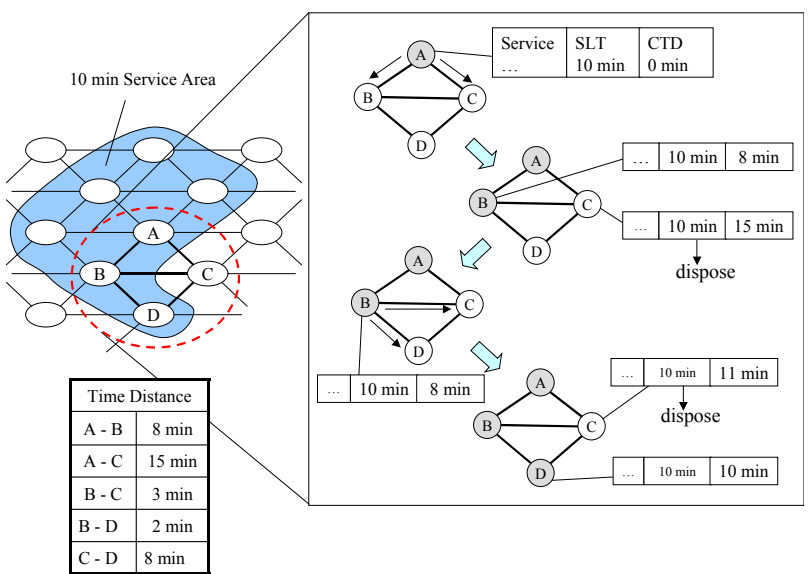

Figure 2: Autonomous Information Provision

In case that the node receives the same request message, which is shown by the same SID, if CTD of the latter message is not smaller than that of the former, the node disposes of it. Otherwise, the node processes it in the same way of the first receiving.

Thus the request message from SP can be provided to all the nodes in the community based on the property of the service lifetime flexibly. In this way, flexibility to provide the information based on service is achieved.

Figure 2 shows the sequence of a service provision. When a SP in node A provides a 10-minute service, each node autonomously judges based on the lifetime and time distance whether it forwards the service to the neighbor nodes. As the result, the service is distributed to the users in appropriate service area, and the flexibility in the service provision is achieved.

\subsection{Process of Collecting Users' Marketing Information}

After providing the request message to the nodes in the community based on the service by autonomous information provision technology, the nodes broadcast the request message to the users in cell.

All the messages from users are sent to SP in the following way. Each user sends his demand information containing SID of the request message to the nearest node, and the node sends it to the upper node that sent the request message containing the smallest time distance of all the neighbor nodes that sent the same SID. The message is sent to the upper node over and over, and by this means the return path is autonomously judged by each node and all the messages from the users are sent to $\mathrm{SP}$ in this system.

\section{Proposed Technologies}

\subsection{Autonomous Synchronization Technique}

\subsubsection{Problem in Collecting Process}

In order to finish collecting all the users' information 
in the community in real time, there are two necessities. SP must receive the users' information soon, and counting process of that must be finished soon.

It takes little time for SP to receive all the users' information by the primitive way that each node receiving the information from users only sends the information to the upper node without any processing as soon as it receives. But counting process only at one node costs much time because there are so many users in the community. In this centralized way, real-time property cannot be achieved.

\subsubsection{Autonomous Synchronization Technique}

In order to achieve real-time property in counting all the users' marketing information in the community, Autonomous Synchronization Technology is proposed.

If only SP does counting process of users' demand information, even if SP receives them as soon as possible, it takes much time. Hence, load sharing among the nodes in the community is necessary to achieve real time property. In order to decrease SP's load, not only SP but also each node in the community has to do collecting process of the users' requirement information. If each node collects the messages of the requirement information and integrates them before sending them to the upper node, the messages received by SP decrease and processing time are both decreased. The messages of user's marketing information are sent asynchronously and continuously from user's mobile devices to the nearest node through the wireless connection. Therefore, each node can get the multiple messages by waiting in a certain period of time and synchronizing them.

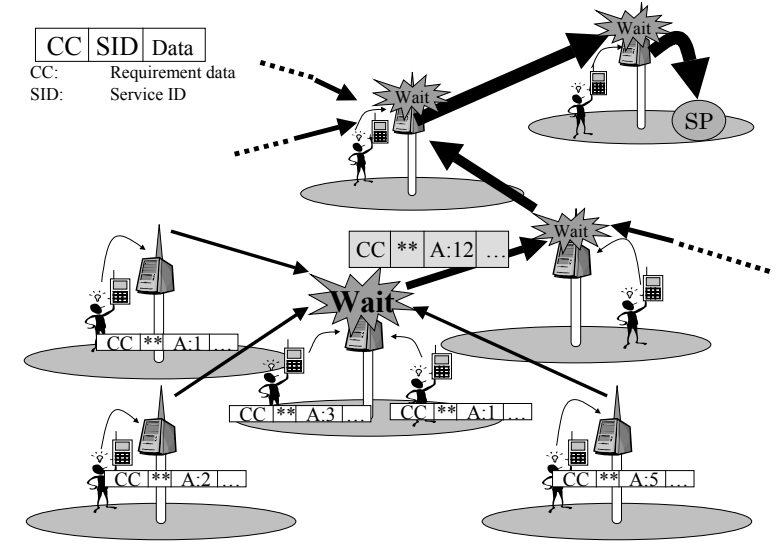

\section{Figure 3: Autonomous Synchronization Technique}

When each node receives the message from a user or a neighboring node, it begins to wait in the synchronizing time to receive the other marketing information. Each node does not need to know which node sends the messages. If the node receives the other messages containing the same SID from the other users or the other neighbor lower nodes in this time, it integrates these messages into one message. It forwards the integrated message to the upper node when the synchronizing time is expired. Each node does not know when a node sends the messages. If the message is received after forwarding the integrated message, the node waits, synchronizes and integrates it with the next receiving messages, and forwards it to the upper node.

The asynchronous messages are synchronized and integrated continuously by each node, and the messages received by the upper nodes including SP are decreased. As the result, the response time that SP finishes collecting process of all the users' demand information is decreased.

\subsection{Autonomous Cooperation Technique}

\subsubsection{Problem in Information Provision}

Autonomous Cooperation Technique is proposed to achieve fault tolerance in the provision of the services. Figure 4 shows an example of fault propagation. Normal case shows the service flow when all the nodes in the service area are operating correctly. When node $\mathrm{C}$ has failed, node $\mathrm{E}$ and $\mathrm{F}$ cannot receive the service. In this case, fault propagation occurs, and the node failure causes the decrease of the reliability of the service distribution. Therefore techniques for achieving fault tolerance in the provision of the services are necessary.

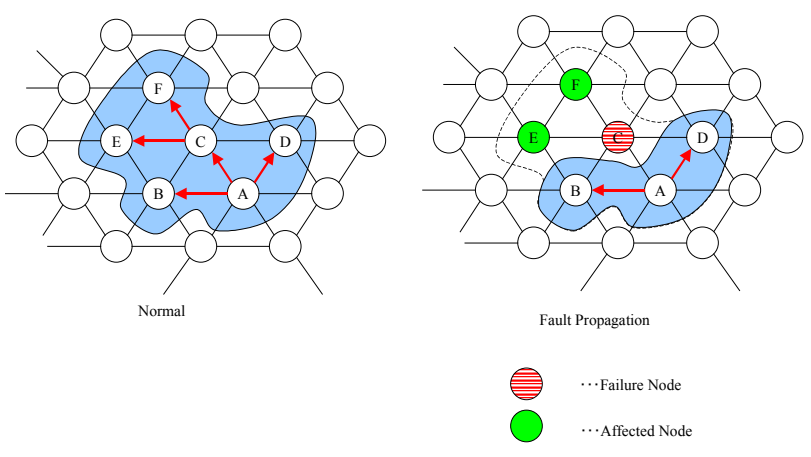

Figure 4: Fault Propagation

\subsubsection{Autonomous Cooperation Technique}

Each node sends a signal to neighboring nodes at regular time intervals $\mathrm{T}$. When a certain node is down, the signal is stopped. The neighboring nodes can detect the node's down by watching the signal. If the signal does not come even if it passes over the time $\mathrm{T}$, the node is judged as a failure node. When the neighboring nodes of the failure node send the message, they attach the urgent header containing urgent flag, failure node ID and sender node ID to the normal message, and send to every neighboring node except the failure node. Nodes, which receive the urgent message, judge whether the message should be sent to the neighboring or disposed. If the node has a failure neighboring, it sent every neighboring node unconditionally and checks the message history and judge whether the cumulative time can be restored. If the cumulative time is restored, the urgent header is removed and the message is sent as a normal message. If it does not have a failure neighboring, the urgent message is 
disposed. Thus, information is surely distributed except failure nodes (Figure 5).

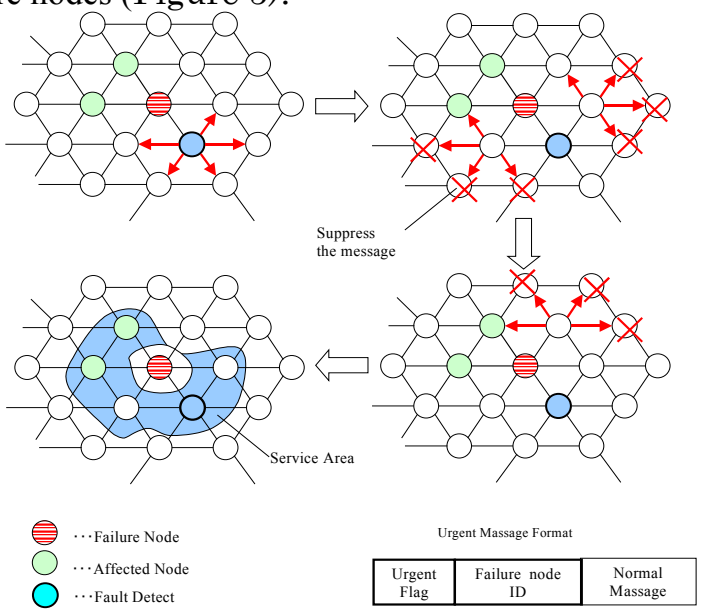

Figure 5: Autonomous Cooperation Technique

During this process, all of the other nodes, as well as the fault detecting node, continue normal operation.

\section{Evaluation}

Proposed technology is evaluated with the following model, and the effectiveness is clarified.

\subsection{Autonomous Synchronization Technique}

\subsubsection{Evaluation Model}

To evaluate the effectiveness of the proposed technology, the response time, which is defined as the time from when SP sends the request message until when SP gets the result of the information collected from all the users' marketing information, is compared with the centralized way that only SP does collecting process.

The assumption of the simulation environment is shown as following.

There is one SP in the network, and a certain number of users distributed equally. Each node has the same capacity and each node can process 500 users' data per second. Each node has 6 neighbor nodes and 250 users in each cell as we assume $100[\mathrm{~m}]$ in cell's radius and Japanese general city's density of population. Each user sends the information randomly. The community area changes like a concentric circle and the number of the users in the community changes as the community area is changing.

\subsubsection{Results}

We compare the response time of the proposed technology with that of the centralized one under the condition that the community area changes from 1 layer ( 1 node, 250 users) to 10 layers (54 nodes, 67750 users).

In Figure 6 there are two cases of the proposed technology where synchronizing time (ST) is 0.3 [s] and 0.1 [s]. The case of $\mathrm{ST}=0.1$ is better than that of $\mathrm{ST}=0.3$. This shows that there exists a relation between synchronizing time and response time. If synchronizing time is small, it takes little time for SP to receive messages but the number of messages received by SP is large and collecting process of SP costs much time. But on the other hand, synchronizing time is large, it takes much time for SP to receive messages but the number of messages received by SP is small and collecting process of SP costs little time.

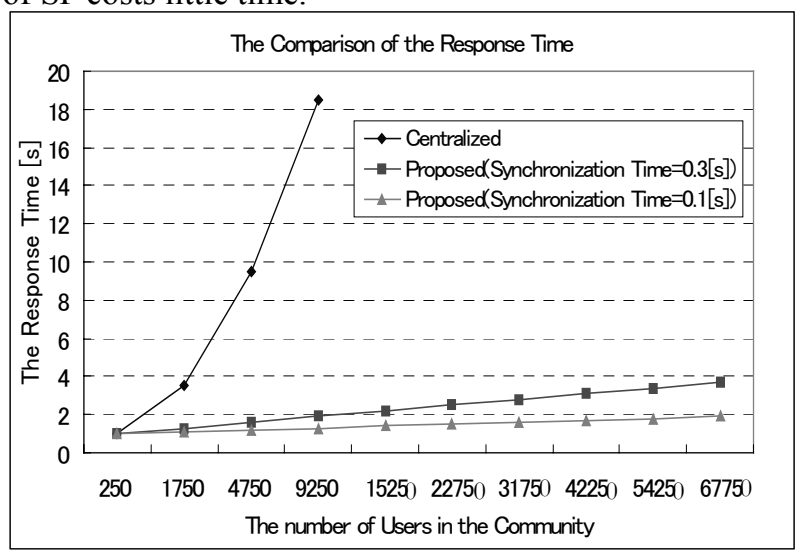

Figure 6: Simulation Results (1)

Figure 7 shows the relation between synchronizing time and response time under the condition that the community has 10 layers, 54 nodes and 67750 users.

It describes that there is an optimal synchronizing time (OST) and it is 0.027 [s]. In the case that ST is less than OST, the response time is increasing rapidly because of load concentration in some nodes. In the case that ST is more than OST, the response time is increasing gradually because of the overhead of waiting time.

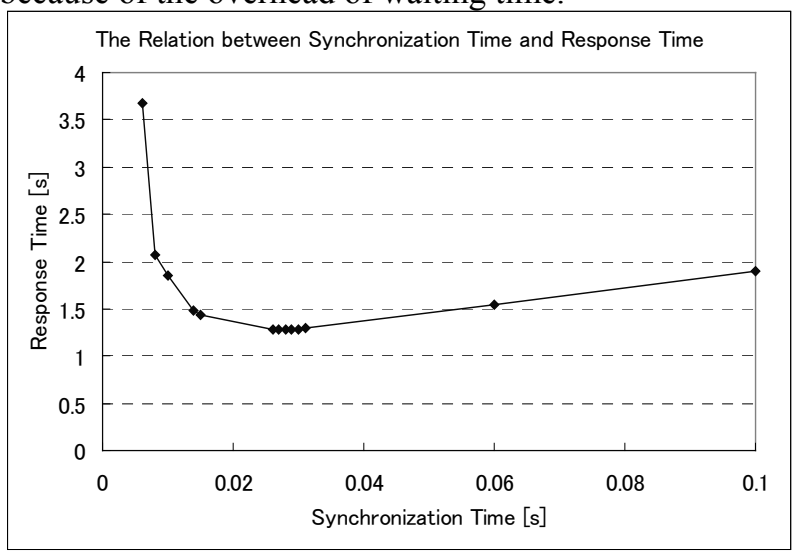

Figure 7: Simulation Results (2)

\subsection{Autonomous Cooperation Technique}

\subsubsection{Evaluation Model}

The reliability of the service distribution $\mathrm{S}_{\mathrm{N}}$ is given as $S_{N}=\frac{n}{N}$

where $\mathrm{N}$ is the total number of nodes, and $\mathrm{n}$ is the number of nodes which is possible to transmit the messages to users in the service area.

We can now compare the service availability of the proposed system with the centralized management systems. For simplicity, and to clarify the difference in 
their reliability of the service distribution, we will assume that the reliability of the centralized server is Rs with a value of 0.95 , and of every node is $R_{n}$ with a value of 0.90 . We will also assume that the reliability of the centralized server decrease with the increase of the total number of the node $\mathrm{N}$ because of the load congestion. These ratio are $\mathrm{T}_{\mathrm{s}}$ with a value of 1.1 and $\mathrm{T}_{\mathrm{n}}$ with a value of 1.01 respectively. $\mathrm{Rs}_{\mathrm{s}}(\mathrm{N})$ represents the reliability of the centralized server with $\mathrm{N}$ nodes, and $\mathrm{R}_{\mathrm{n}}(\mathrm{N})$ shows the reliability of the nodes.

For the Centralized system, $\mathrm{S}_{\mathrm{N}}$ is given by the following equation:

$$
S_{N}=R_{S}(N) \sum_{k=0}^{N}{ }_{N} C_{k}\left(1-R_{n}(N)\right)^{k}\left(R_{n}(N)\right)^{(N-k)}
$$

The number of the working nodes $\mathrm{N}$ for the proposed system is given by the following equation:

$$
S_{N}=\sum_{k=0}^{N}{ }_{N} C_{k}\left(1-R_{n}(N)\right)^{k}\left(R_{n}(N)\right)^{(N-k)}
$$

\subsubsection{Results}

Figure 8 represents the reliability of the service distribution. The result shows that the reliability of the centralized system is high rate when the system consists of few nodes, but the reliability of the proposed system is high rate when the number of the nodes increases.

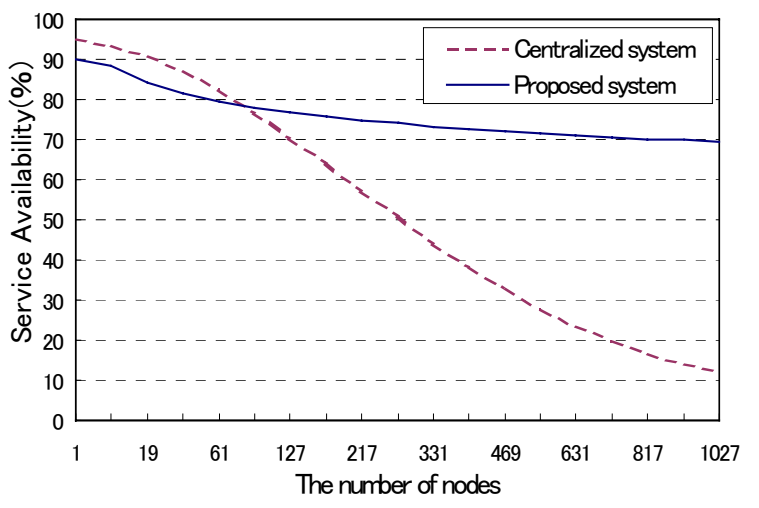

Figure 8: Simulation Results (3)

\section{Conclusions}

It has been increasing even more that the requirement for mobile commerce to provide the timely services reflecting the users' preference under the evolving market. Users would like to utilize the appropriate services reflecting their location, time and preference. SP would like to grasp the current users' requirements in the area based on each service at the moment to determine the effective service.

In order to satisfy both the users and SP's requirements, Service Oriented Community is proposed. In the Community, the members cooperate with each other in order to get mutual benefits like social community. SP grasps the current requirements by collecting users' marketing information in the community area based on services in real time. Furthermore, the users and the SP would like to utilize and provide stable services.

Time Distance has been proposed as the efficient measure of the community area, and Time Distance Oriented Service System has been proposed to achieve flexibility to provide users' marketing information and SP's services based on their time property.

Here, Autonomous Synchronization Technique and Autonomous Cooperation Technique are proposed to achieve real-time property and fault tolerance respectively. By using Autonomous Synchronization Technique, each node waits for the multiple messages from users in the cell and neighboring nodes, integrates them into one message, and forwards to the upper node over and over. As a result, the number of messages received by $\mathrm{SP}$ is decreased, as the result the time to finish collecting process at SP is decreased. By Autonomous Cooperation Technique, each node autonomously monitors the neighbor nodes. When the node detects the neighbor nodes' failure, it starts to send the urgent message and other nodes autonomously judge whether they forward it to their neighbor nodes or not. As the result, the services are surely provided to the nodes within service area.

Effectiveness of the proposed technologies is clarified. It assures real-time property and fault tolerance even in the case of increasing the number of users.

\section{References}

[1] K. Mori, "Autonomous Decentralized System: Concept, Data Field Architecture and Future Trends", Proc. of ISADS93, pp28-34, March 1993.

[2] K. Mori, S. Yamashita, H. Nakanishi, et al., "Service Accelerator (SEA) System for Supplying Demand Oriented Information Services", Proc. of IEEE 3rd ISADS, pp.129-136, April 1997.

[3] ADSS DSIG (Autonomous Decentralized Service Systems, Domain Special Interest Group), White Paper for ADDS, ads/98-12-01, OMG, 1997, http://www.omg.org/

[4] J. Hightower and G. Borriello, "Location Systems for Ubiquitous Computing," IEEE Computer, vol.34, no.8, pp.57-66, 2001.

[5] N. Marmasse and C. Schmandt, "Location-Aware Information Delivery with ComMotion," HUC2000, LNCS1927, pp.157-171, 2000.

[6] Y-C. Tseng, W-H. Liao and C-M. Chao, "Location Awareness in Ad-Hoc Wireless Mobile Networks," IEEE Computer, vol.34, no.6, pp.46-52, 2001.

[7] Takanori Ono, Khaled Ragab, Naohiro Kaji and Kinji Mori, "Service Oriented Communication Technology for Achieving Assurance," 1st International Workshop on Assurance in Distributed Systems and Networks, 2002. 